\title{
Plasmonic Scattering as an Efficient Tool for a Force-Free Technique to Follow Single DNA Molecules
}

\author{
Moshe Lindner ${ }^{*}$, Guy Nir and Yuval Garini \\ Department of Physics and the Institute for Nanotechnology, Bar Ilan University, Ramat-Gan 52900, Israel
}

\begin{abstract}
One of the promising methods for single molecule studies is Tethered Particle Motion (TPM). The technique layout that was developed a decade ago [1], is based on anchoring one end of a DNA molecule (or any other polymer of interest) to a surface, and labeling the opposite end with an optical marker. In solution, the marker moves randomly in a volume that is governed by the restrictions set by the DNA molecule. Tracking and analyzing its position distribution provides an essential tool to follow the dynamics of the DNA conformations [2, 3]. Most of the TPM systems use a CCD camera to detect the projected position of the bead on a two-dimensional plane, and the information on the bead height above the surface is lost. Here we show how TPM can be exploited for three-dimensional particle tracking using Total Internal Reflection (TIR) [4] illumination system. We also report of the deviations between the lateral distribution and the axial distribution.
\end{abstract}

Keywords: DNA, single molecule, tethered particle motion, total internal reflection microscopy.

\section{INTRODUCTION}

During the last few decades, many efforts were made to provide new methods for single molecule studies in order to characterize biological processes with a high spatial and temporal resolution [5]. Single-molecule studies enable one to characterize molecular processes and identify physical, chemical, or biological sub-populations that are not accessible through ensemble studies and identify rare states. Common ensemble studies provide only limited information on the possible heterogeneity in a group of single molecules. In contrast, single molecule data avoid large population averaging and sub-populations can therefore be identified and quantified, thereby leading to new insights that are otherwise inaccessible [6].

One of the most intriguing subjects in biology today is the study of DNA-protein interactions that govern genome stability [7] and regulate gene expression [8]. Many of the well-known methods, e.g. magnetic or optical traps, involve the use of external force which stretches $[9,10]$ or twists $[11$, 12] DNA during biological processes. These applied forces might alter protein-DNA binding probabilities and interfere with the nature of some processes, especially due to the fact that DNA is normally found in a condensed conformation [13]. TPM overcome this, since no external forces applied to the sample [14].

\section{DNA End-to-End Distribution}

One can think of DNA as a chain of short segments that are tilted with respect to one another at a relative small angle, that still have some statistical distribution. Therefore, if the orientation of a certain segment along the DNA is known, the orientation of its neighboring segment will be

*Address correspondence to this author at the Department of Physics and the Institute for Nanotechnology, Bar Ilan University, Ramat-Gan 52900, Israel; Fax: +972-3-7384054; Tel: +972-3-5317393; E-mail: moshe.lindner@gmail.com almost the same. However, for a segment which is far away, the orientation is unknown since the small angles along the chain are summed. The distance at which the orientation correlation is lost is defined as the persistence length, $l_{p}[2$, 15]. It depends on the structure of the DNA (or polymer in general) and on the environmental conditions of the solution, such as the $\mathrm{pH}$ level. Interestingly, this simple model of the DNA allows one to calculate the expected distance between the ends of the DNA by modeling it as a random walk process [13]. The solution for a one-dimensional end-to-end distribution is a one-dimensional Gaussian:

$P_{1 D}(x) d x=\sqrt{\frac{3}{4 \pi L l_{p}}} \cdot \exp \left(-\frac{3 x^{2}}{4 L l_{p}}\right) d x$

where $L$ is the contour length (i.e. the length of a fully stretched polymer) of the DNA and $l_{p}$ is the persistence length.

The distribution for a two-dimensional projection is the multiplication of $P(x) \cdot P(y)$ and in polar coordinates it gives the Rayleigh distribution:

$$
P_{2 D}(R) d R=2 \pi R \cdot \frac{3}{4 \pi L l_{p}} \cdot \exp \left(-\frac{3 R^{2}}{4 L l_{p}}\right) d R
$$

where $R$ is the projected end-to-end distance on a two dimensional plane.

In a TPM experiment, one end of the DNA is tethered to a surface, so the distribution in the lateral $x y$ plane is given by the above equations. On the other hand, the distribution along the axial $(z)$ axis is different than equation (1). The difference arises because the motion along the axial direction is limited by the surface; nor the bead, nor any part of the polymer can penetrate it, which leads to a different distribution. The axial distribution is given by the following function which is the difference of two Gaussians $[16,17]$ : 


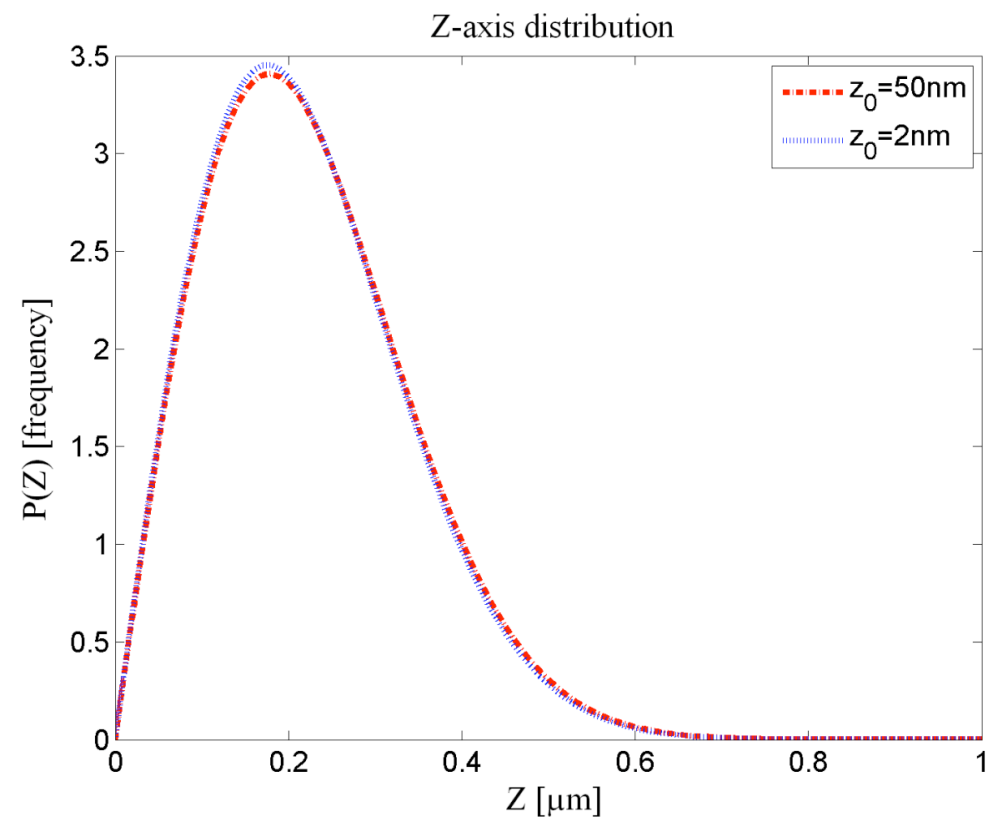

Fig. (1). Theoretical distribution of the DNA end along the z-axis for tethered DNA. Two distributions are shown according to equation (3), but a different parameter $z_{0}$ is used. Notice that it has a negligible influence on the distribution.

$P(z) d z=\frac{1}{\Omega}\left[\exp \left(-\frac{3\left(z-z_{0}\right)^{2}}{4 l_{p} L}\right)-\exp \left(-\frac{3\left(z+z_{0}\right)^{2}}{4 l_{p} L}\right)\right] d z$

where $\Omega=\sqrt{\frac{\pi 4 l_{p} L}{3}} \cdot\left[1-\operatorname{erfc}\left(\frac{3 z_{0} / l_{p} L}{2 \sqrt{3 / l_{p} L}}\right)\right]$ is the normalization factor, and $z_{0}$ is a short length that is in the range of the width of DNA $(2 \mathrm{~nm})$ and the persistence length $(50 \mathrm{~nm})$. Its exact value has negligible influence on the distribution, as shown in Fig. (1). Notice that this function is similar to the Rayleigh function that is described above (equation 2) for the radial planar distribution, but these two equations results from different reasons. While the origin of equation (2) is the multiplication of a Gaussian function and the unit-area as defined in polar coordinates, equation (3) results from a onedimensional random walk near an impenetrable surface.

Several attempts to measure the distribution $P(z)[18,19]$ did not clearly follow equation (3) due to the specific experimental setup parameters that were used (bead radius, contour length and polymer type), and showed normal distribution.

\section{Experimental Method}

As explained above, TPM is based on anchoring one end of the studied DNA molecule to a surface, while its other end is attached to a marker. The size of the marker must be small relative to the DNA end-to-end distance. Otherwise, the position of the bead will be mainly dominated by its own size, and not by the positions that are allowed by the DNA. This issue was treated theoretically and resulted in a criterion that should be fulfilled for proper experiments. It is based on the definition of a parameter named the excursion number, $N_{R}=r / \sqrt{L \cdot l_{p} / 3}$, which must be smaller than one, where $r$ is the marker radius [20]. As one can see, the marker radius should be small enough to fulfill this criterion. On the other hand, the position of the bead must be determined with high precision, which usually means that a rather large bead is needed. To overcome these contradicting requirements, we used a small metal bead together with a dark-field microscopy setup. Due to plasmonic effects, the scattering from such a bead is relatively intense and it is higher than that of a polystyrene bead of the same size [5]. This high intensity results from Rayleigh scattering:

$$
I \propto \frac{r^{6}}{\lambda^{4}}\left|\frac{(m / n)^{2}-1}{(m / n)^{2}+2}\right|^{2}
$$

where $r$ is the bead radius, $\lambda$ is the vacuum wavelength and $m$ and $n$ are the complex indexes of refraction of the bead and the medium respectively. For metals, the imaginary part of $m$ has a high value in the visible spectrum [21], and hence the intensity is high.

We studied $\sim 925 \mathrm{~nm}$ long double-stranded DNA molecules, and as a marker we used gold nano-beads with a diameter of $80 \mathrm{~nm}$. It gives for the excursion number a value of $N_{R} \approx 0.32$ which is small and shows that the bead size does not affect the measured distribution. The DNA is attached to a passivated surface using digoxigenin (DIG) anti-DIG linker, and the bead is attached to the DNA with biotin - anti-biotin linker (Fig. 2).

In order to track the beads in the axial $z$-direction we used TIR illumination through an equilateral prism (Fig. 2) that is attached to a fluidic cell with immersion oil to ensure a refractive index matching. When the illumination light hits the glass-water surface at an angle which is above the critical angle, $\theta_{c}=\sin ^{-1}\left(n_{t} / n_{i}\right)$, it generates an evanescent electromagnetic field with intensity that decreases exponentially with the distance from the surface $[22,23]$ : 


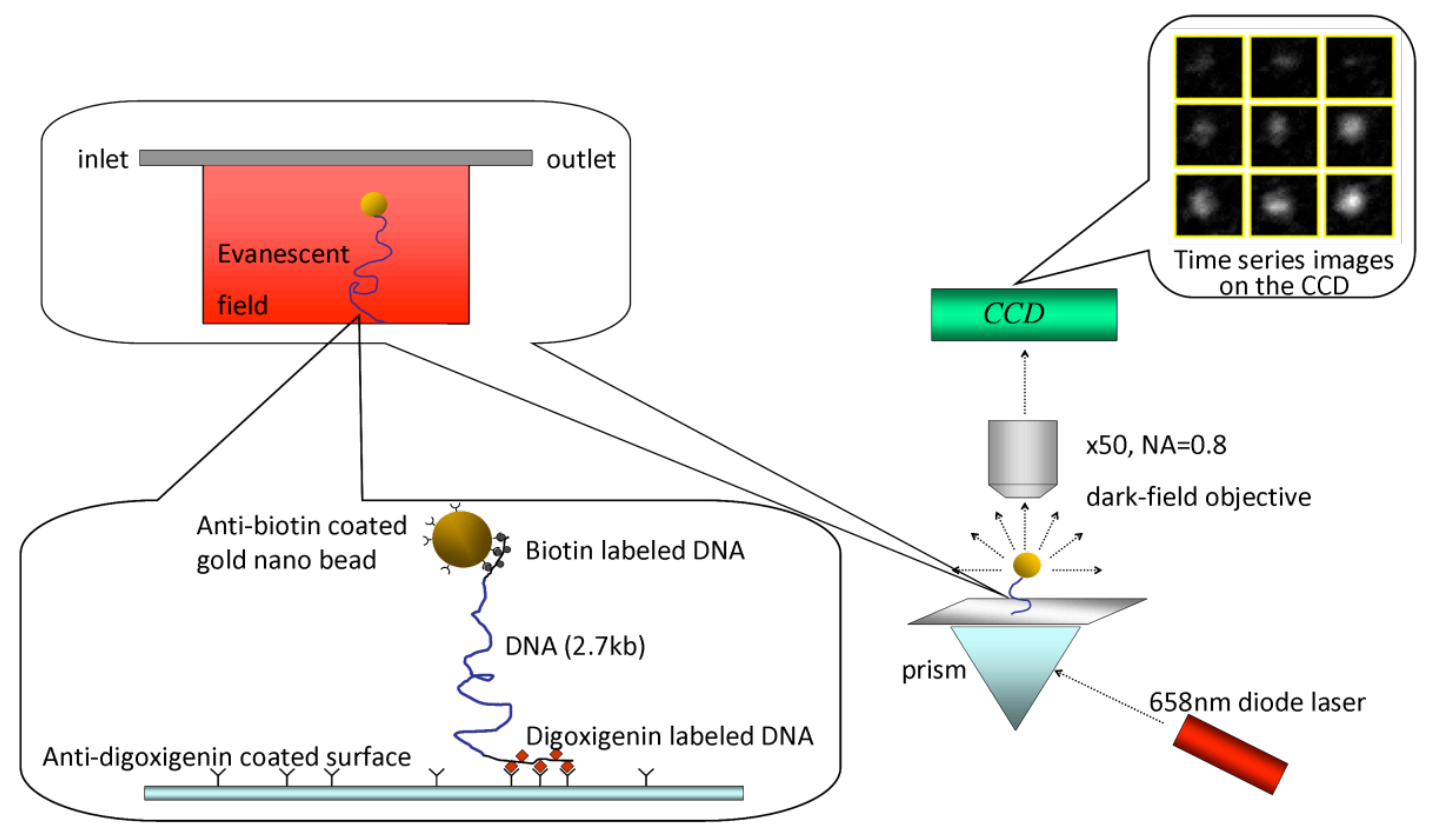

Fig. (2). The experimental setup. Gold nano bead is attached to one end of DNA, while its other end is attached to the surface. The total internal reflection setup uses a prism that is attached to the glass with immersion oil to match the indexes of refraction. The incident angle of the laser on the prism is selected in order to create an evanescent field with a relatively large penetration depth. The imaging system includes a x50 darkfield objective-lens with a numerical aperture of 0.8 and a sensitive EM-CCD camera (see text for more details).

$I(z)=I_{0} e^{-z / d}$

where $d$ is the penetration depth:

$d=\frac{\lambda}{4 \pi \sqrt{n_{i}^{2} \sin ^{2} \theta_{i}-n_{t}^{2}}}$

In our setup $n_{i}=1.5$ (glass) and $n_{i}=1.3$ (water), so $\theta_{c} \approx 60^{\circ}$. As we are interested to measure the bead position also at distances which are few hundreds of nanometer above the surface, we chose a rather long wavelength $(658 \mathrm{~nm}$ diode laser), and we tuned the angle to be very close to the critical angle, so the penetration depth is relatively large. In this angle, the light hit the prism perpendicularly, and the losses of intensity in the air-glass interface are minimized.

The scattering from the bead was collected using a $\mathrm{x} 50$ dark-field [24] objective lens $(\mathrm{NA}=0.8)$ and recorded with an EM-CCD camera which is sensitive and allows to use a gain factor that improves the signal to noise ratio, especially with respect to the read-out noise of the CCD. The sensitivity of the camera is high, so that relatively short exposure times of 1-3 ms can be used. The exposure time is also an important parameter. During the exposure time, the moving bead scans a growing area, which leads to an error in finding the bead position. Therefore, the bright signal of the bead together with the sensitive camera allows to decrease the smearing effect significantly. Even with the short exposure times we use, the smearing effect still narrows the measured distribution with respect to the real one, and we therefore use a correction function [25] to extract the right values.

Typically, 1500-3000 frames are collected and provide enough statistical information for extracting the relevant information. Then, the position of the bead and its intensity were extracted using MATLAB program that we have written based also on a DIPimage library (www.diplib.org).

The lateral position is calculated using center of mass algorithm [19] with a precision of few nanometers [26], and the axial position is determined by the light intensity using the relation: $z(t)=-d \ln [I(t)]$, where $d$ is the penetration depth of the evanescent field and $I$ is the light intensity. Finding $d$ is crucial for a precise determination of the bead height, and requires an adequate calibration procedure [27]. Nevertheless, here we only show the distribution along $z$ in order to test the type of distribution, and therefore the exact calibration is not crucial.

\section{RESULTS AND DISCUSSION}

Fig. (3) shows a typical experimental data for the bead distribution along the in-plane radial axis $R$ and along the axial direction perpendicular to the surface $z$. The planar distribution (left) was fitted to the Rayleigh distribution (equation 2) with a single fitting parameter, the persistence length, that was found to be $l_{p}=47 \pm 2 \mathrm{~nm}$ in agreement with typical values that are reported in the literature [28]. The fitting is quite good, with coefficient of determination $r^{2}=0.96$.

The $z$-axis distribution (Fig. 3 right) was measured as explained above from the intensity of the bead at each frame. The graph therefore shows the distribution of the bead as a function of its height above the surface. We found the distribution to be as predicted by theory (equation 3 ). The coefficient of determination from the fit gave a value of $r^{2}=0.978$. 

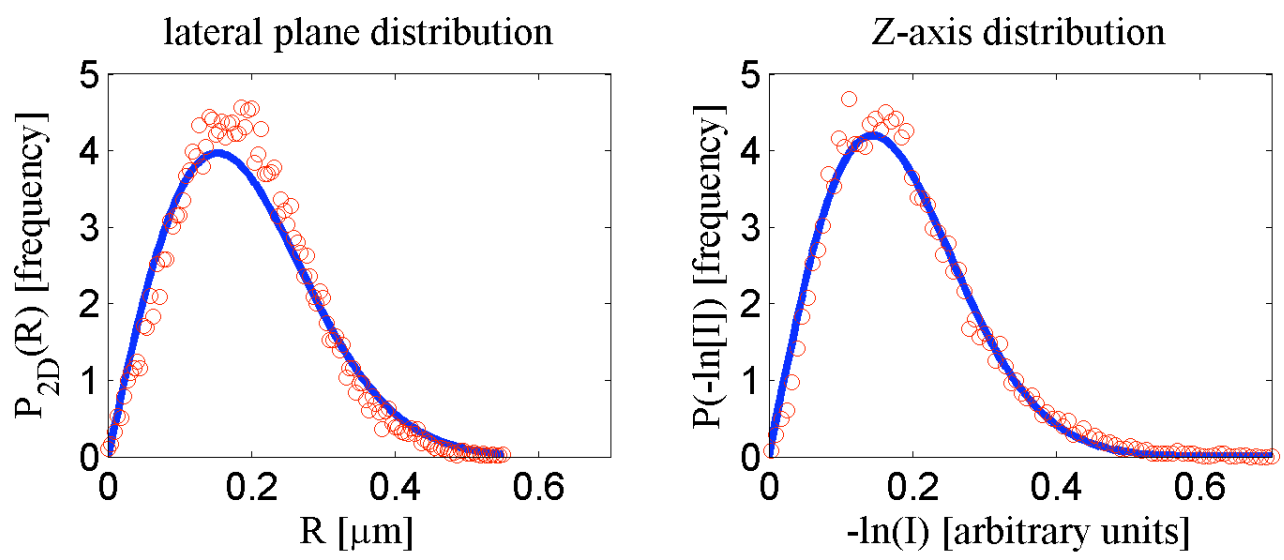

Fig. (3). Experimental results. Left: lateral distribution (circles) and fit to equation (2) (solid line). It allows to extract the persistence length of the DNA which is found to be $l_{p}=47 \pm 2 \mathrm{~nm}\left(r^{2}=0.96\right)$. Right: logarithm of the light intensity distribution (circles). It is proportional to the $z$-axis distribution (but requires to find the penetration depth $d$ ), and the fit to equation (3) (solid line). A good fit is found ( $r^{2}=0.978$ ), demonstrating the validity of the theoretical prediction.

Notice that we did not calibrate the penetration depth of the evanescent field, and hence we do not show the real $z$ axis distribution. Nevertheless, the only difference between the data we show and the real $z$-axis distribution is a scaling factor that does not change the shape of the distribution.

The measured Rayleigh-like distribution results only from the number of possible configurations of the DNA at any possible height, and it is not affected by interaction with the surface which is assumed to be zero. It shows that the tail of the DNA is not found close to the surface, but actually relatively far from it. Such a configuration may have important implications for processes that involve the DNA configuration near surfaces, such as the conformations that the DNA adopts in the nucleus of cells.

\section{CONCLUSIONS}

We demonstrate a method to measure and analyze the three-dimensional distribution of a metal nano-bead in a TPM experiment. A fast particle tracking can be applied with a relatively simple optical setup that does not require a confocal microscope setup [29]. The data acquisition for the $z$-axis can be even faster, since only the intensity is relevant, and it can be measured using a fast PMT detector instead of CCD camera.

Using the method we measured the distribution along the $z$-axis, and showed its agreement with theory. Although the theory is known for decades, it was never measured before in a way that can clearly be compared to the theory as shown here.

The distribution of the z-axis shows that the bead is very rarely found near the surface, but at a significant height above it, and according to the theory given above, the peak of the probability is found at a height of $\sim 180 \mathrm{~nm}$ above the surface. This behavior can be observed as a repulsion of the DNA end from the surface, even though no external force is applied and the effect is solely a result of the interactions of the entropy of the DNA in the solution and the boundary. This repulsion may be important in biological systems that involve the DNA interactions and may lead to better understanding of DNA organization in the cell.

\section{ACKNOWLEDGEMENT}

We thank Yitzhak Rabin, Shay Rappaport and Shlomi Medalion for their help in the theoretical aspects of this work. This work was supported in part by the Israel Science Foundation, Grants No. 985/08, No. 1729/08, No. 1793/07 and No. 25/07.

\section{CONFLICT OF INTEREST}

None declared.

\section{REFERENCES}

[1] Schafer DA, Gelles J, Sheetz MP, Landick R. Transcription by single molecules of RNA polymerase observed by light microscopy. Nature 1991; 352: 444-8.

[2] Lindner M, Nir G, Dietrich HRC, et al. Studies of single molecules in their natural form. Isr J Chem 2009; 49: 283-91.

[3] Dietrich HRC, Young IT, Garini Y. Gold nanoparticles: A novel application of spectral imaging in proteomics - preliminary results. Proc SPIE 2005; 5694: 82-9.

[4] Axelrod D, Burghardt TP, Thompson NL. Total internal reflection fluorescence. Annu Rev Biophys Biol 1984; 13: 247-68.

[5] Selvin PR, Ha T. Single-molecule techniques: a laboratory manual. Cold Spring Harbor Laboratory Press 2008.

[6] Walter NG, Huang C-Y, Manzo AJ,Sobhy MA. Do-it-yourself guide: how to use the modern single-molecule toolkit. Nat Meth 2008; 5: 475-89.

[7] Higgins NP. The bacterial chromosome. Hardcover, ASM Press 2004.

[8] Elf J, Li G-W, Xie XS. Probing transcription factor dynamics at the single-molecule level in a living cell. Science 2007; 316:1191-4.

[9] Grier DG. A revolution in optical manipulation. Nature 2003; 424: 810-6.

[10] Wang MD, Yin H, Landick R, Gelles J, Block SM. Stretching DNA with optical tweezers. Biophys J 1997; 72: 1335-46.

[11] Deufel C, Forth S, Simmons CR, Dejgosha S, Wang MD. Nanofabricated quartz cylinders for angular trapping: DNA supercoiling torque detection. Nat Meth 2007; 4: 223-5.

[12] Van Der Heijden T, Modesti M, Hage S, et al. Homologous recombination in real Time: DNA strand exchange by RecA. Mol Cell 2008; 30: 530-8. 
[13] Rubinstein M, Colby RH. Polymer physics. USA: Oxford University Press 2003.

[14] Nir G, Lindner M, Dietrich HRC, et al. HU protein induces incoherent dna persistence length. Biophys J 2011; 100: 784-90.

[15] Gutjahr P, Lipowsky R, Kierfeld J. Persistence length of semiflexible polymers and bending rigidity renormalization. Europhys Lett 2006; 76: 994.

[16] Chandrasekhar S. Stochastic problems in physics and astronomy. Rev Mod Phys 1943; 15: 1.

[17] Slutsky M. Diffusion in a half-space: From Lord Kelvin to path integrals. Am J Phys 2005; 73: 308-14.

[18] Jensenius H, Zocchi G. Measuring the spring constant of a single polymer chain. Phys Rev Lett 1997; 79: 5030 .

[19] Blumberg S, Gajraj A, Pennington MW, Meiners J-C. Threedimensional characterization of tethered microspheres by total internal reflection fluorescence microscopy. Biophys J 2005; 89: $1272-81$.

[20] Segall DE, Nelson PC, Phillips R. Volume-exclusion effects in tethered-particle experiments: bead size matters. Phys Rev Lett 2006; 96: 0883061-4.

[21] Johnson PB, Christy RW. Optical constants of the noble metals. Phys Rev B 1972; 6: 4370.
[22] Prieve DC, Walz JY. Scattering of an evanescent surface wave by a microscopic dielectric sphere. Appl Opt 1993; 32: 1629-41.

[23] Prieve DC, Frej NA. Total internal reflection microscopy: A quantitative tool for the measurement of colloidal forces. Langmuir 1990; 6: 396-403.

[24] Braslavsky I, Amit R, Ali BMJ, et al. Objective-type dark-field illumination for scattering from microbeads. Appl Opt 2001; 40: 5650-7.

[25] Wong WP, Halvorsen K. The effect of integration time on fluctuation measurements: Calibrating an optical trap in the presence of motion blur. Opt Express 2006; 14: 12517-31.

[26] Pouget N, Dennis C, Turlan C, et al. Single-particle tracking for DNA tether length monitoring. Nucl Acids Res 2004; 32: e73 1-7.

[27] Lindner M, Nir G, Medalion S, et al. Force-free measurements of the conformations of DNA molecules tethered to a wall. Phys Rev E 2011; 83: 011916.

[28] Phillips R, Kondev J, Theriot J. Physical biology of the cell. Garland Science 2008.

[29] Lehner R, Koota J, Maret G,Gisler T. Segment Distributions of End-Tethered Polymers in a Good Solvent. Phys Rev Lett 2006; 96: 107801.

Received: May 15, 2011

Revised: October 05, 2011

Accepted: November 13, 2011

(C) Lindner et al.; Licensee Bentham Open.

This is an open access article licensed under the terms of the Creative Commons Attribution Non-Commercial License (http:/creativecommons.org/ licenses/by-nc/3.0/), which permits unrestricted, non-commercial use, distribution and reproduction in any medium, provided the work is properly cited. 